\title{
Primary Central Nervous System Lymphoma: Diffuse Large B-Cell Lymphoma
}

\author{
Primer Santral Sinir Sistemi Lenfoması: Diffüz Büyük B Hücreli Lenfoma
}

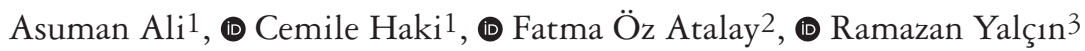

1 University of Health Sciences, Yuksek Ihtisas Training and Research Hospital, Clinic of Neurology, Bursa, Turkey

2Uludag University Faculty of Medicine, Department of Pathology, Bursa, Turkey

3Burtom Radiological Diagnostic Center, Bursa, Turkey

Keywords: Diffüz büyük B hücre lenfoması, beyin manyetik rezonans görüntüleme, stereotaktik beyin biyopsisi

Anahtar Kelimeler: Diffuse large B-cell lymphoma, brain magnetic resonance imaging, stereotactic brain biopsy

\section{Dear Editor,}

A 56-year-old female patient was first evaluated by the psychiatry clinic with symptoms of nausea and vomiting and altered mental status such as disorientation, empty speech, impulse control disorder. The patient was then referred to the neurology clinic because the clinical picture did not change with psychiatric treatment. Progressive somnolence, confusion, dysphagia, dysphonia, and left hemiparesis were added to the clinical picture. One week after her admission to the clinic, she had a generalized epileptic seizure that started focally on the left side. Brain magnetic resonance imaging (MRI) (1.5 Tesla) and cerebrospinal fluid analysis were performed. Brain MRI (Figure 1, 2, 3) revealed multiple heterogeneous lesions with irregular contrast involvement covering a large part of the right frontoparietal lobe, extending along the white matter pathways, continuing to the right part of the pons, and affecting the corpus callosum, bilateral thalamus, right basal ganglia and left anterior frontal lobe. Mega-dose methyl prednisolone was administered intravenously for ten days. Her somnolence decreased, the mental state disorder disappeared, and she started to walk independently. However, although her wellbeing continued for thirteen days, the previous clinical picture recurred. The methylprednisolone treatment was reapplied but the patient only partially benefited. MRI spectroscopy (Figure 4) was performed, and $\mathrm{N}$-acetyl aspartate levels decreased and choline and lipid peaks increased. Perfusion MRI findings also suggested a low vascular tumor. Stereotactic brain biopsy was performed, and histopathologic and immunohistochemical results were consistent with large B-cell lymphoma infiltration. In the preparations stained with hematoxylin and eosin, widespread, large-sized lymphoid infiltration with narrow cytoplasm, small number of nucleoli, and coarse chromatin structure was detected. T-cell markers were stained negatively, B cell markers and CD20 were strongly positive. Ki67 index was high (70\%). With these features, the patient was diagnosed as having CD20-positive diffuse large B-cell lymphoma. No involvement was detected in lymphoid and non-lymphoid areas on the other side of the body, and treatment was planned considering a primary central nervous system lymphoma (PCNSL). The patient underwent 180 cGy curative whole-brain radiotherapy and was followed up. Somnolence, brainstem findings, and left hemiparesis persisted and there was no change in the clinical picture. Seven days after radiotherapy, her breathing was impaired and she became comatose. She died during intensive care follow-up.

PCNSLs are responsible for $0.7-1.7 \%$ of malignant lymphomas (1). In individuals without immunodeficiency, they are diagnosed between the ages of 45-70 years and the average incidence is around 50 years. Seizures are less common than other types of brain tumors (2). Our patient also had a single seizure that did not recur. Perfusion MRI and MRI spectroscopy (Figure 4) are used in clinical radiology practice to differentiate central nervous system lymphomas from other brain lesions $(2,3,4)$. In our case, the maximum relative cerebral blood volume was typically lower

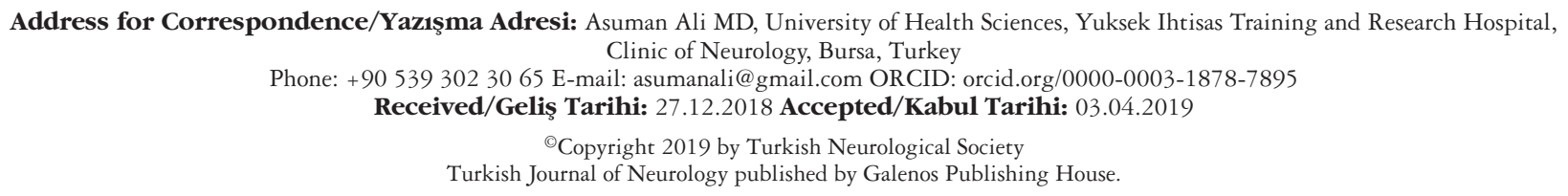



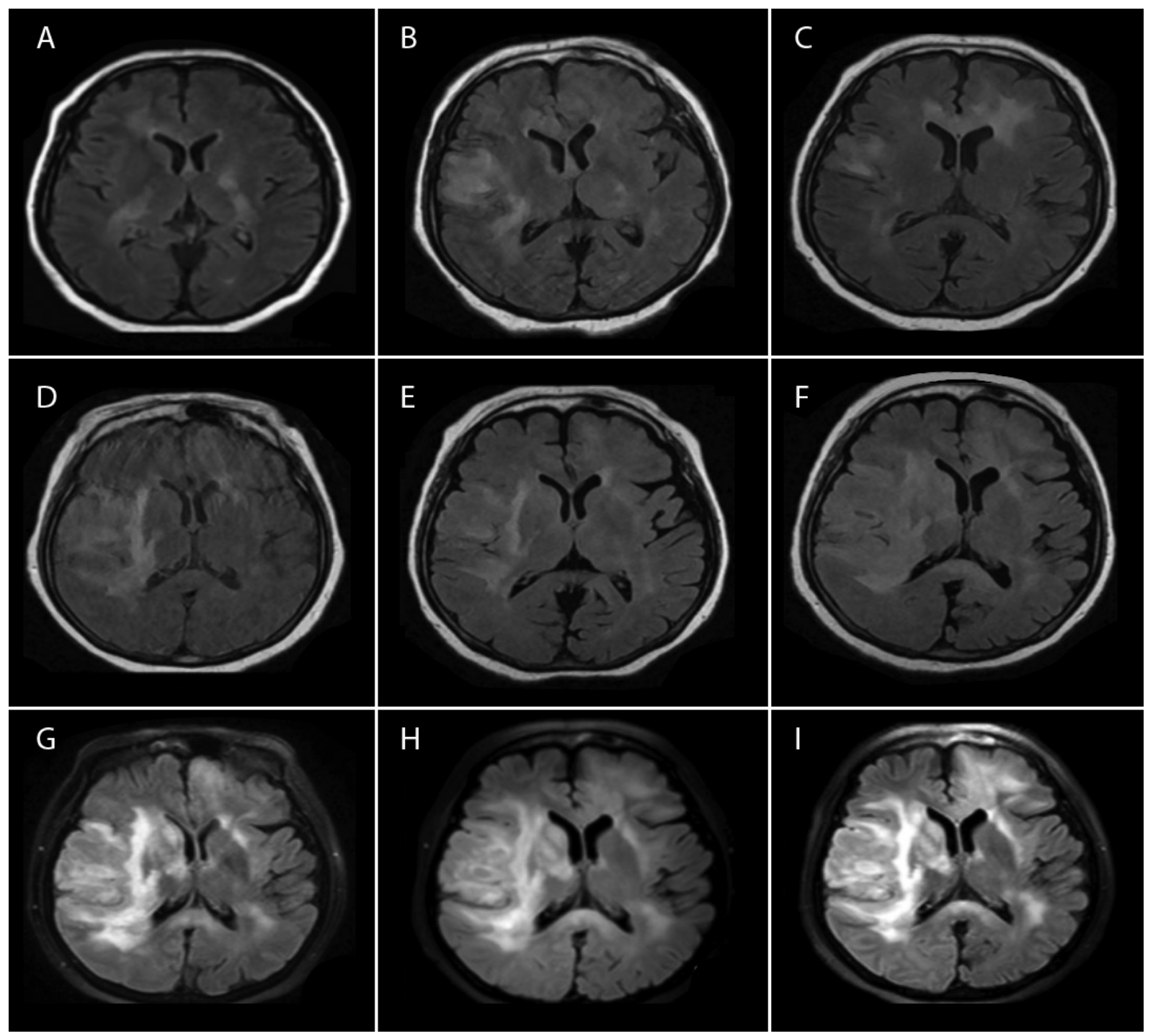

Figure 1. A) T2 FLAIR images. B) Scattered, unrelated spread in the right frontal deep white matter, right thalamic region, right medial temporal, genu of the corpus callosum, left thalamic region and brain stem when the lesion was first detected. C) Forty days later, the lesion became more widespread and the right frontal lobe was more affected. D, E, F, G, H, I) A partial regression was observed in the control after fifty-four days

in tumor tissue in perfusion MRI. The most common areas of involvement are frontal lobes followed by basal ganglia and the thalamus. Frontal lobe localization is reported as $20-43 \%$, whereas basal ganglia involvement is reported as $13-20 \%$. Brain stem or cerebellum or both are affected in $9-13 \%(2,4)$. In our case, the frontal lobe was bilaterally but asymmetrically affected, and basal ganglion and thalamus involvement were also observed.
Brain lesions in PCNSL may mimic multiple sclerosis, and clinical signs and symptoms usually respond to corticosteroids (5). Therefore, stereotactic biopsy is necessary for the definitive diagnosis and accurate definition of the lesion. Whole-brain radiotherapy is the first effective treatment option because PCNSLs are usually multifocal but radiosensitive. 

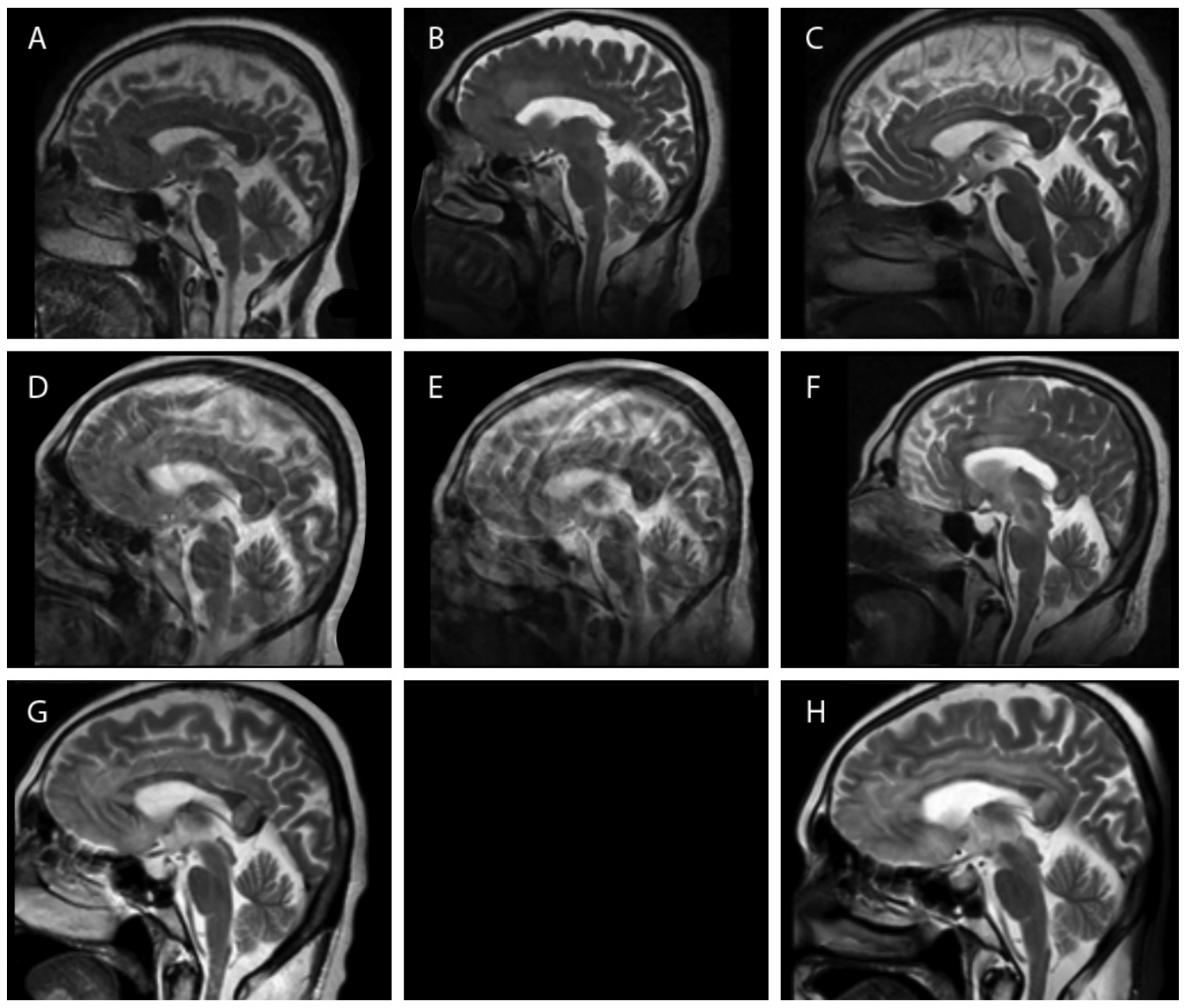

Figure 2. A) T2 sagittal images. B, C, D, E, F, G, H) When the lesion was first detected, the genu of the corpus callosum was involved, but later, it was observed that the lesion affected the entire corpus callosum and spread to the pericallosal area in serial follow-ups up to 40-125 days. No regression was observed at 70, 80, 105, 115, 120, 125 days after the initial detection of the lesion and a rapid spread was observed
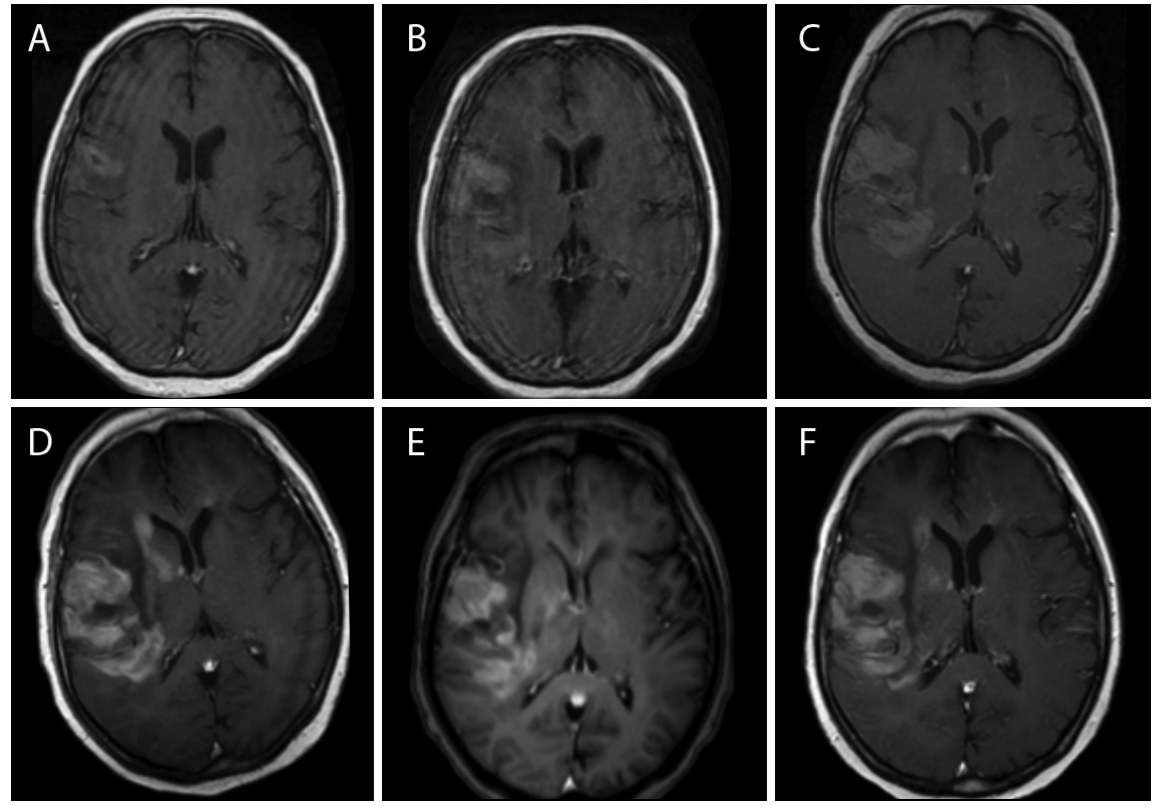

Figure 3. A) T1 contrast-enhanced axial images. Although partial enhancement was observed only in the right frontal areas 54 days after the initial detection of the lesion, B, C, D, E, F) the lesion became widespread with increased enhancement during the follow-ups up to 80-125 days 


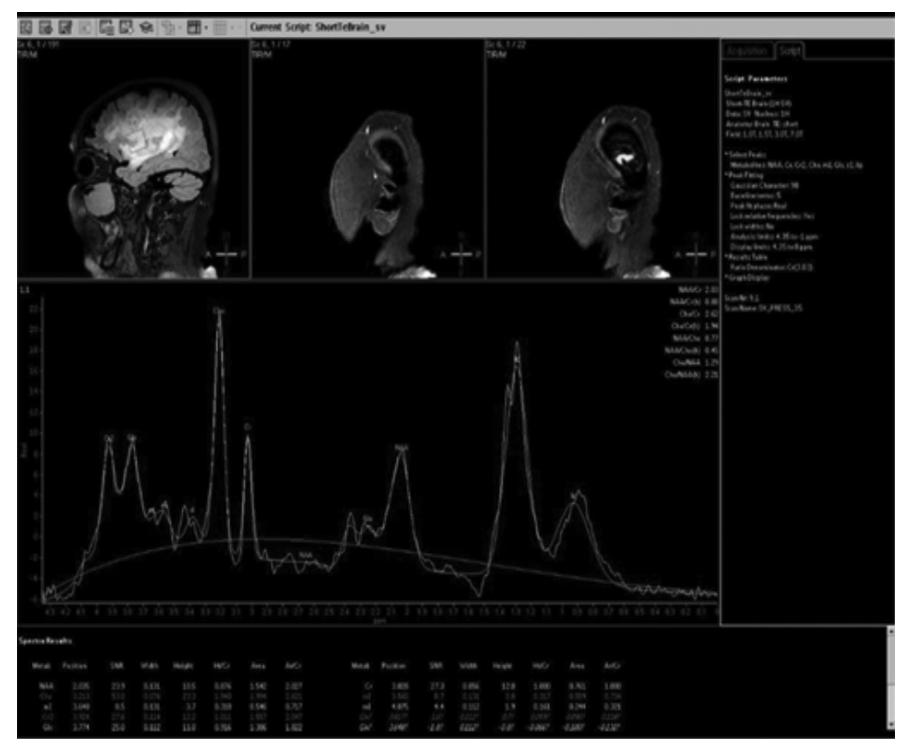

Figure 4. Magnetic resonance imaging spectroscopy. In the spectroscopic study from the right frontal lobe, suppression of $\mathrm{N}$-acetyl aspartate value and a significant increase in choline and lipid peaks were observed in the lesion area

\section{Ethics}

Informed Consent: The consent has been received from the partner of the patient.
Peer-review: Internally peer-reviewed.

\section{Authorship Contributions}

Surgical and Medical Practices: A.A., R.Y., Concept: A.A., Design: A.A., Data Collection or Processing: A.A., F.Ö.A., R.Y., Analysis or Interpretation: A.A., F.Ö.A., Literature Search: A.A., C.H., Writing: A.A.

Conflict of Interest: No conflict of interest was declared by the authors.

Financial Disclosure: The authors declared that this study received no financial support.

\section{References}

1. Brar R, Prasad A, Shama T, Vermani N. Multifocal lateral and fourth ventricular B-cell primary CNS lymphoma. Clin Neurol Neurosurg 2012;114:281-283.

2. Nabazivadeh S, Vossough A, Hajmomenian M, Assadsangabi R, Mohan S Neuroimaging in Central Nervous System Lymphoma. Hematol Oncol Clin N Am 2016;799-821.

3. Zhang S, Li H, Zhu R, Zhang M. Application value of magnetic resonance imaging in diagnosing central nervous system lymphoma. Pak J Med Sci 2016;32:389-393.

4. Senocak E, Karlı Oğuz K, Ozgen B, et al. Parenchymal lymphoma of the brain on initial MR imaging: A comparative study between primary and secondary brain lymphoma. E J R 2011;79:288-294.

5. Kvarta MD, Sharma D, Castellani RJ, et al. Demyelination as a harbinger of lymphoma: a case report and review of primary central nervous system lymphoma preceded by multifocal sentinel demyelination. Bio Med Central Neurol 2016;16;72;1-7. 